\title{
Rythme circadien de l'adénylyl cyclase dans l'épiphyse
}

L'épiphyse, ou glande pinéale, joue un rôle majeur en chronobiologie (alternance veille/sommeil ; reproduction) en produisant la mélatonine pendant la nuit exclusivement. Les oscillations nycthémérales des taux circulants de la mélatonine sont le message biologique de la photopériodicité. La mélatonine, une fois produite, diffuse à l'extérieur des pinéalocytes en traversant passivement la membrane plasmique. L'hormone n'étant ni stockée, ni activement libérée, la régulation circadienne de ses taux circulants résulte des étapes enzymatiques qui contrôlent sa synthèse. Dans les cellules de la glande pinéale, le précurseur de la mélatonine, la sérotonine, est dans un premier temps acétylé en $\mathrm{N}$-acétylsérotonine par la sérotonine-N-acétyltransférase (NAT) pour être ensuite converti en mélatonine sous l'action catalytique de la hydroxyindole-O-méthyltransférase (HIOMT) [1].

On sait, depuis les années 1970, que la synthèse circadienne de la mélatonine est sous le contrôle périodique de la noradrénaline provenant des afférences sympathiques. Ces afférences, qui transmettent des informations de la rétine, libèrent la noradrénaline uniquement pendant la nuit. Deux types de récepteurs adrénergiques sont présents dans la membrane plasmique des cellules de la glande pinéale: (1) les récepteurs $\beta$, dont l'activation augmente le taux de l'AMP cyclique et (2) les récepteurs $\alpha$ l qui sont couplés à la phospholipase C et aux systèmes de signalisation intracellulaire dépendants du $\mathrm{Ca}^{2+}$. Les deux récepteurs sont impliqués dans la régulation de la synthèse
L'activité enzymatique de la NAT augmente considérablement pendant la nuit $\left(\mathrm{m} / \mathrm{s} n^{\circ} 3\right.$, vol. $\left.12, p .381\right)$. Selon des travaux datant d'il y a plus de quinze ans, la NAT est étroitement contrôlée par le taux d'AMP cyclique du fait de sa phosphorylation par la protéine kinase A (PKA), l'effecteur par excellence de l'AMP cyclique [2].

Depuis, des oscillations circadiennes de plusieurs molécules-clés de la signalisation cellulaire ont été observées dans la glande pinéale. Elles concernent le facteur transcriptionnel CREB ( $c A M P$ responsive element binding protein), dont le taux de phosphorylation augmente pendant la nuit, et toute une série de facteurs de transcription induits par CREB. Ainsi, il a été démontré que l'expression de ICER (inducible cAMP early repressor) dans la glande pinéale obéit à une régulation circadienne. Ce membre de la famille CREM (cAMP responsive element modulator) réprime la transcription dépendante de l'AMP cyclique et est exprimé uniquement pendant la nuit [3-5]. De plus, on a montré que le promoteur du gène codant pour la NAT comporte des séquences CRE (cyclic AMP responsive elements) et que son $\mathrm{ARNm}$ varie de façon circadienne selon le même schéma que l'ARNm de ICER $[4,6]$ Un vrai puzzle de régulations circadiennes semble donc se former autour de l'axe de synthèse de la mélatonine. La noradrénaline libérée pendant la nuit induit une élévation du taux de l'AMP cyclique qui déclencherait non seulement l'induction (transcriptionnelle et post-transcriptionnelle) de la NAT, mais aussi l'induction de ICER qui opérerait comme répresseur du système. Le sys- tème de l'AMP cyclique jouerait, par conséquent, un rôle essentiel dans la synchronisation des signaux intracellulaires.

La capacité du système de l'AMP cyclique d'intégrer les différents messages intracellulaires varie-t-elle entre le jour et la nuit? Et dans ce cas, cela est-il dû à une oscillation circadienne d'un élément propre à ce système? Afin de repondre à ces questions, nous avons entrepris une analyse du système enzymatique de synthèse de l'AMP cyclique [7] dans la glande pinéale à différents moments du nycthémère. Nous avons démontré que l'expression de l'adénylyl cyclase du type 1 se fait suivant un strict profil circadien [8]. L'ARNm de l'AC 1 est maximal pendant le jour et très faiblement exprimé pendant la nuit. De plus, on a observé une augmentation massive de la sensibilité de l'enzyme au $\mathrm{Ca}^{2+}$ pendant la nuit, ce qui tendrait à faire penser que le niveau de la protéine enzymatique est maximal pendant la nuit. Cela peut être attribué au délai entre l'expression de l'ARNm et la synthèse de la protéine fonctionnelle. L'expression de l'AC 1 est sous le contrôle de la noradrénaline et de la lumière environnante. Bien qu'on ne puisse pas affirmer qu'il s'agisse là d'une régulation transcriptionnelle, l'oscillation circadienne du taux de l'ARNm de l'AC 1 est en faveur d'un mécanisme régulateur à long terme de l'activité du système de l'AMP cyclique. Par ailleurs, le fait que l'AC 1 soit aussi activée par le complexe calcium/calmoduline, suggère que plusieurs neurotransmetteurs agissant par le calcium pourraient aussi intervenir dans la synthèse de l'AMP cyclique, et par 
conséquent dans la production de la mélatonine. Il s'agirait là d'un mécanisme homéostatique très important qui permettrait à la glande pinéale de s'adapter à l'environnement photopériodique en fonction d'une multiplicité de signaux simultanés.

E.T.

J.H.
1. Reiter RJ. Pineal melatonin : cell biology of its synthesis and of its physiological interactions. Endoc Rev 1991; 12 : 151-80.

2. Buda M, Klein DC. A suspension culture of pinealocytes: regulation of $\mathrm{N}$-acetyl transferase activity. Endocrinology 1978; 103: 1483-93.

3. Stehle JH, Foulkes NS, Molina CA, Simmoneaux $\mathrm{V}$, Pevet P, Sassone-Corsi P. Adrenergic signals direct rythmic expression of transcriptional repressor CREM in the pineal gland. Nature 1993; 365: 314-20.

4. Sassone-Corsi P. Le gène CREM et les bases moléculaires de l'horloge biologique. médecine/ sciences $1993 ; 9$ : 1253-5.
5. Mick G, Jouvet M. Rythmes circadiens: leurs bases anatomiques, fonctionnelles et moléculaires. médecine/sciences 1995; 11:52-61.

6. Borjigin J, Wang MM, Snyder SH. Diurnal variation in mRNA encoding serotonin $\mathrm{N}$-acetyltransferase in pineal gland. Nature 1995; 378: 783-5.

7. Hanoune J. Les adénylyl cyclases des mammifères. médecine/sciences $1994 ; 10: 444-7$.

8. Tzavara E, Pouille Y, Def er N, Hanoune J. Diurnal variation of the adenylyl cyclase type 1 in the rat pineal gland. Proc Natl Acad Sci USA 1996; 93 : 11208-12.

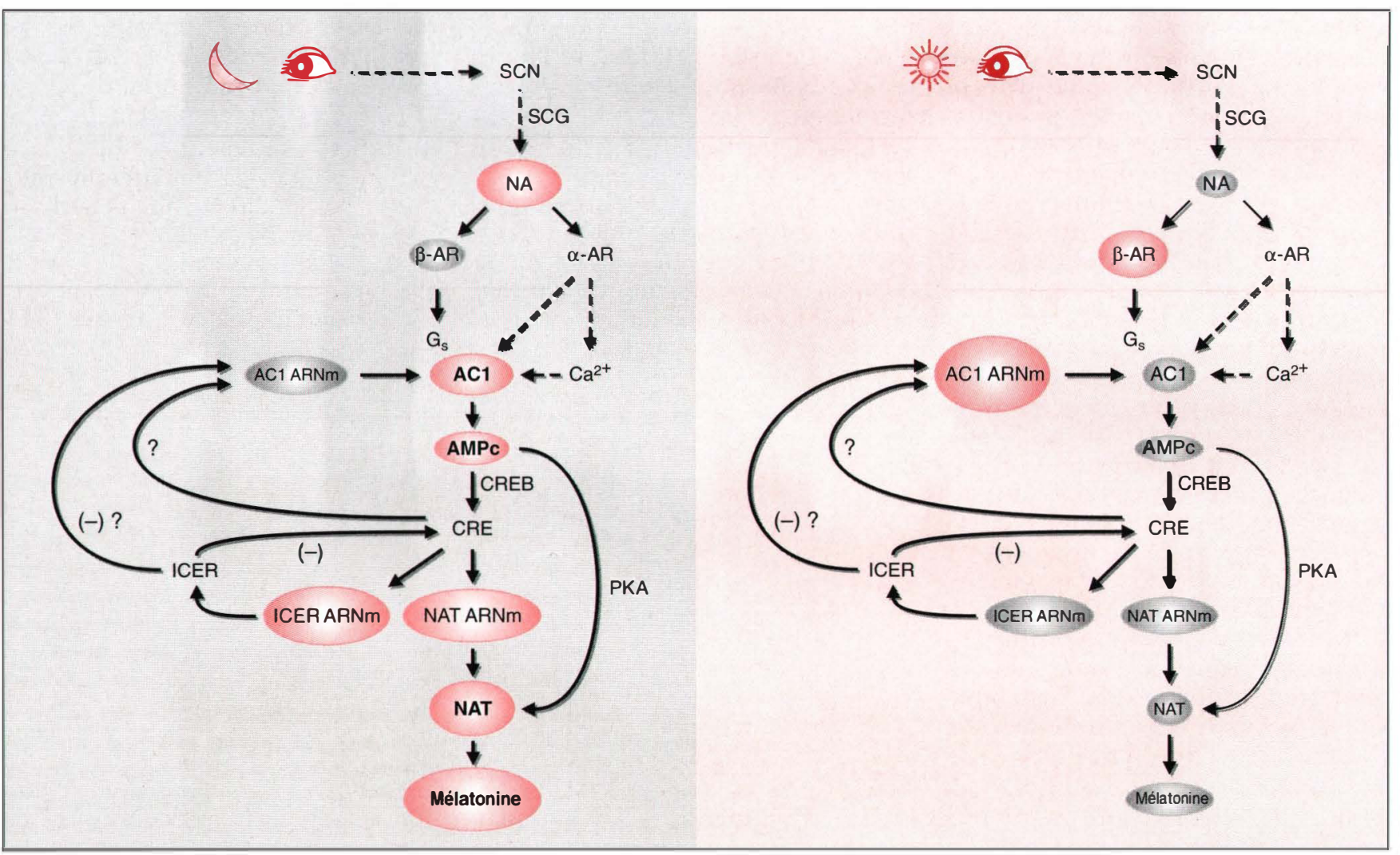

Figure 1. Régulations circadiennes des étapes contrôlant la synthèse de la mélatonine dans l'épiphyse. Le message rythmique lumière/obscurité arrive à la glande pinéale par l'intermédiaire d'une voie multisynaptique dont font partie la rétine, le noyau suprachiasmatique (SCN) et les ganglions cervicaux supérieurs (SCG). Pendant la nuit, la noradrénaline (NA) libérée des SCG stimule les récepteurs $\beta$ et $\alpha 1$-adrénergiques qui activent l'adénylyl cyclase de type I (AC1) par le biais, respectivement, des protéines $G$ et du $\mathrm{Ca}^{2+}$. Pendant le jour, l'expression de l'ARNm de l'AC1 atteint son maximum. Cela conduit à une augmentation de la concentration de la protéine fonctionnelle pendant la nuit, vu le délai nécessaire à sa synthèse. L'élévation nocturne de la concentration d'AMP cyclique induit une augmentation de l'activité enzymatique de la N-acétyl transférae (NAT), et donc de la production de mélatonine. En outre, I'AMP cyclique stimule l'expression de ICER (inducible cAMP early repressor), dont le produit réprime la transcription dépendante de l'AMP cyclique en formant ainsi une boucle d'autorégulation dans laquelle pourrait s'inscrire la régulation de la synthèse de l'AC1. 